\title{
A free action for pions as quark composites
}

\author{
Sergio Caracciolo \\ Scuola Normale Superiore and INFN - Sezione di Pisa \\ I-56100 Pisa, ITALIA \\ Internet: Sergio.Caracciolo@sns.it \\ Fabrizio Palumbo * \\ INFN - Laboratori Nazionali di Frascati \\ P. O. Box 13, I-00044 Frascati, ITALIA \\ Internet: palumbof@lnf.infn.it
}

September 18, 1997

\begin{abstract}
In the framework of an approach to bosonization based on the use of fermionic composites as fundamental variables, a quadratic action in even Grassmann variables with the quantum numbers of the pions has been constructed. It includes the $\sigma$-field in order to be invariant under $[S U(2)]_{L} \otimes[S U(2)]_{R}$ transformations over the quarks. This action exhibits the Goldstone phenomenon reducing its symmetry to the $\mathrm{O}(3)$ isospin invariance. A squared mass for the pions is generated according to PCAC.

The model has been investigated in the Stratonovitch-Hubbard representation, in which form it is reminiscent of the Gell-Mann-Lévy model. By the saddle point method a renormalizable expansion in inverse powers of the index of nilpotency of the mesonic fields (which is 24), is generated. The way it might be used in a new perturbative approach to QCD is outlined.
\end{abstract}

*This work has been partially supported by EEC under TMR contract ERB FMRXCT96-0045 


\section{Introduction}

Recently it has been suggested that the correlation functions of fermionic composites can be evaluated by a change of variables whereby the composites themselves are assumed as integration variables. Although the constituents cannot be expressed in terms of the composites, we can talk about a generalized change of variables in a precise sense [1].

In the context of QCD the motivation is to set up a perturbative expansion in terms of the hadrons instead of the usual one in terms of the quarks. A prerequisite for this goal is the existence of a free action for the composites. Surprisingly enough, for trilinear composites like the barions the free action turns out to be the Dirac one. This is due to the fact that if the trilinear composites are chosen in the proper way, their integral is identical to the Berezin integral over the constituents [1].

The integral over even composites instead does not reduce in general to a Berezin or to an ordinary integral, and therefore there is no reason why their free action should resemble that of free bosons or fermions. Indeed we remind the reader that Grassmann variables $\phi$ are characterized by the index of nilpotency, which is the smallest integer $\Omega$ such that

$$
\phi^{n}=0, \text { for } n>\Omega \text {. }
$$

The index of odd composites is always 1 (and this is related to the simplicity of their action), while the index of even composites depends on their structure: For the pions, for instance, it is 24. The action of even composites is therefore expected to be, on general grounds, a polynomial of degree $\Omega$, and this expectation is confirmed in the cases where it has been derived. There are some results in the strong coupling limit of lattice QCD [2], (also at finite density [3]) because in that limit the composites appear as the natural starting point of an expansion. In a similar spirit in numerical simulations there have been efforts to replace the direct evaluation of the fermionic determinants by the so-called monomer-dimer expansion [4].

All these actions are defined on a lattice and have a common feature : They are static in the formal continuum limit. Moreover, if the Grassmann field is replaced by a true bosonic field, they are unbounded from below.

Our first reaction against such a result was to attribute it to the strong coupling regime, which is generally thought to be intrinsically far from the continuum. But this explanation has been ruled out by the investigation of a simple quadratic action for a Grassmann scalar of index 1 [5]. In this work 
there was no attempt to relate such an action to that of constituents, but only to see whether it was possible to obtain a sensible propagator in the continuum limit. The result was, indeed, that the action should be static (in this limit) and negative definite, in which case it is possible to obtain for the propagator a random-walk representation.

To get further insight it was necessary to obtain in an exact way a free action for composites starting from that of the constituents. For this reason a simple nonrelativistic model was considered, the so-called pairing model, where the program of the use of even Grassmann variables for the description of composites has been successfully completed [6]. Here the composite is a Cooper pair which has index $\Omega=j+\frac{1}{2}, j$ being the angular momentum of the fermions involved ( see Sec. 2 ). Again the action for these composites is a polynomial of degree $\Omega$ with the properties described above, which where then conjectured to characterize all even composites.

While this result confirms the specific features previously found, it is rather discouraging, because a derivation of the composite action along the lines of the pairing model appears to be exceedingly difficult in relativistic field theories, and in any case the resulting action too difficult to deal with. Its complexity, however, appears due to the fact that it must reproduce the two-point functions of all the nonvanishing powers of the composites

$$
\left\langle\left[\phi^{*}(x) \phi(y)\right]^{n}\right\rangle, \text { for } n \leq \Omega .
$$

But in Particle Physics we are essentially interested in the correlation functions of the first power of the composites (namely with $n=1$ in (1.2), to be referred to in the sequel as the $n=1$ correlation functions), and under this restriction the effective action might be much simpler. In Sec. 2, in fact, we investigate whether a quadratic action can yield these two-point functions for the pairing model, and we find an affirmative answer.

We can then explore a short cut in the determination of an effective action for even composites, based on the fact that such an action, due to its high dimension, can be added to that of the constituents by only altering by a finite amount the renormalization group parameters. One should proceed in the following way: first determine, if it exists, a quadratic free action for the mesons, and then check in a perturbative calculation if such an action can absorb, by appropriate renormalizations, the contributions from the free action of the constituents. The present paper is devoted to the first part of this program, but our results can also be regarded, in a more modest perspective, just as the construction of a model. 
In Sec. 3 we consider on a lattice a quadratic action for the pions with the general features discussed above. We must mention, however, that we have also found a quite different action of composites which yields a Klein-Gordon propagator, but it gives rise to a nonrenormalizable expansion.

The propagator with an action quadratic in even Grassmann variables is not in general the inverse of the wave operator, and it is not easy to work out. Therefore by using the Stratonovitch-Hubbard representation [7] we replace the Grassmann variables by ordinary bosonic ones, at the price of dealing with an action which is no longer polynomial. We investigate its properties by means of the saddle point method, which generates a $1 / \Omega$ expansion which, in view of the rather high value of $\Omega$ for the pion, appears quite reasonable.

Furthermore, it is possible to choose the parameters in such a way that a free action, with Klein-Gordon propagators, arises in the continuum limit.

The action we have studied contains the pions as well as the $\sigma$ fields, and it is $[S U(2)]_{V} \otimes[S U(2)]_{A}$ invariant. This symmetry is broken spontaneously to the $O(3)$ isospin symmetry, with massless pions and the expectation value of the $\sigma$ field as order parameter. In the presence of an explicit breaking linear in $\sigma$, the pions acquire a squared mass proportional to the breaking parameter. Results of this kind have already been derived in many ways, but what is new here is that they are produced by a simple quadratic action of composite fields (which can hopefully be used as the unperturbed term in a new perturbative approach to QCD).

Before ending this Introduction we notice that the formalism we are going to develop finds its easiest application in the cases where the action of the composites need not be derived from that of the constituents, like in models where composite fields are introduced since the beginning. The present investigation was indeed originally motivated by such a model of composite gauge fields [1]. Another application has been done in a recent work where the possibility has been considered that the quarks might have lower scaling dimensions 9]. This possibility is excluded for the leptons by the request of unitarity, but such a request is much less stringent for the quarks because of confinement. If we assume for the quarks scaling dimension $1 / 2$, for instance, the hadrons acquire naturally the appropriate dimensions, so that their free action has dimension four and can be introduced since the very beginning in the fundamental action along with that of the quarks. The perturbative proof of unitarity in the hadron sector rests therefore on the existence of a consistent free action for the hadronic composites (a condition, however, which is obviously not sufficient). 


\section{A quadratic action for the pairing model}

The pairing model is a simplified version of the BCS model, which describes a system of fermions living in an energy level of given angular momentum $j$ and interacting pairwise only when the partners are coupled to zero angular momentum. Such a model is a schematic representation of the physics of atomic nuclei far from closed shells where collective excitations associated with a spin zero composite, the Cooper pair, occur. From the point of view of field theory, it is a one dimensional model, the infinitely many spatial degrees freedom being frozen to the components of the angular momentum, which can be considered as intrinsic degrees of freedom.

The euclidean action of the pairing model defined on a lattice of spacing $a$ is

$$
S_{\lambda}=-a \sum_{x=0}^{N-1} \sum_{m=-j}^{j}\left[\bar{\lambda}_{m}(x) \nabla \lambda_{m}(x)\right]-E \bar{\lambda}_{m}(x) \lambda_{m}(x-1)+g \phi^{*}(x) \phi(x-1),
$$

where

$$
\nabla \lambda(x)=\frac{1}{a}[\lambda(x)-\lambda(x-1)]
$$

$\lambda_{m}$ is the Grassmann variable associated to a nucleon of spin $j$ and third component of the spin equal to $m, E$ the nucleon excitation energy, and $g$ the pairing coupling. Notice that in nonrelativistic models the time derivative is non symmetric, so that the problem of the doubling does not occur. Finally $\phi$ and $\phi^{*}$ are the Cooper pair composite fields

$$
\phi=\sum_{m=\frac{1}{2}}^{j}(-1)^{j-m} \lambda_{-m} \lambda_{m}, \quad \phi^{*}=\sum_{m=\frac{1}{2}}^{j}(-1)^{j-m} \bar{\lambda}_{m} \bar{\lambda}_{-m}
$$

which have index of nilpotency $\Omega=j+1 / 2$. The partition function is given by the Berezin integral

$$
Z_{\lambda}=\int[d \bar{\lambda} d \lambda] \exp \left(-S_{\lambda}\right)
$$

where

$$
[d \bar{\lambda} d \lambda]=\prod_{x=0}^{N-1} d \bar{\lambda}_{-j}(x) \ldots d \bar{\lambda}_{j}(x) d \lambda_{j}(x) \ldots d \lambda_{-j}(x)
$$


In the evaluation of the correlation functions of the Cooper pairs we can assume $\phi$ as integration variable replacing the Berezin integral over the nucleon field according to

$$
\int d \lambda_{j} \ldots d \lambda_{-j} \phi^{n}=\int d \phi \phi^{n}=\Omega ! \delta_{n, \Omega} .
$$

It can then be shown that, as far as these correlation functions are concerned, the partition function can be written

$$
Z_{C}=\int\left[d \phi^{*}\right][d \phi] \exp \left(-S_{C}\right), \quad[d \phi]=\prod_{x=0}^{N-1} d \phi(x) .
$$

The Cooper pair euclidean action appearing in the above equation is

$$
\begin{aligned}
S_{C} & =-\sum_{x=0}^{N-1}\left[g \phi^{*}(x) \phi(x-1)+\right. \\
& \left.\ln \left\{\sum_{r=0}^{\Omega} \frac{1}{r !}\left[\phi^{*}(x)\right]^{r} \sum_{s=0}^{r}\left(\begin{array}{l}
r \\
s
\end{array}\right) \frac{(\Omega-s) ![\Omega-(r-s)] !}{(\Omega !)^{2}} \eta^{2(r-s)} \phi^{s}(x) \phi^{r-s}(x-1)\right\}\right]
\end{aligned}
$$

with

$$
\eta=1-a E \text {. }
$$

Notice that the nucleon field must satisfy antiperiodic boundary conditions, so that the $\phi$-field satisfies the periodic ones. We see that this action has nothing to do with the action of true bosons and it is static in the formal continuum limit.

The expansion of the ln generates a polynomial of degree $\Omega$. This polynomial has been evaluated for a few values of $\Omega$, and it has always been found to have the form

$$
S=-\sum_{n=0}^{\Omega} \sum_{x=0}^{N-1} \alpha_{n}\left\{\left[\phi^{*}(x) \phi(x)\right]^{n}+\eta^{2 n}\left[\phi^{*}(x) \phi(x-1)\right]^{n}\right\},
$$

where the $\alpha_{n}$ are numerical coefficients. This form is remarkable because it is similar to that of the mesonic action occurring in the strong coupling limit of QCD, characterized by the absence of terms like $\left(\phi^{*}(x)\right)^{m}(\phi(x))^{m-n}(\phi(x-$ 1) $)^{n}, m \neq n$, due to non trivial cancellations.

In this paper we are only interested in the free part (obtained by putting $g=0$ ), whose correlation functions in the continuum limit are

$$
\lim _{a \rightarrow 0}\left\langle\left[\phi^{n}\left(x_{2}\right) \phi^{*}\left(x_{1}\right)\right]^{n}\right\rangle=e^{-\left(t_{2}-t_{1}\right) n 2 E},
$$


where $t_{k}=a x_{k}$ are the physical times. As said in the Introduction, it is natural to think that if we restrict ourselves to the $n=1$ two-point correlation functions for the first power of the fields, we can generate them by a much simpler action, quadratic in the fields, of the form

$$
S=-\sum_{x, y=0}^{N-1} \phi^{*}(x) A_{x, y} \phi(y)
$$

where $A$ is minus the wave operator which for the sake of convenience is parametrized according to

$$
A=(\alpha+\beta) 1-\beta a \nabla \text {. }
$$

In the Appendix we have evaluated the correlation functions for the above action, with the result

$$
\left\langle\left[\phi^{*}(0) \phi(x)\right]^{n}\right\rangle=\left\{\sum_{k=0}^{\Omega} f_{k}^{N}\right\}^{-1} \frac{1}{\beta^{n}} \sum_{k=0}^{\Omega-n} \frac{(k+n) !}{k !}\left(f_{k+n}\right)^{x+1}\left(f_{k}\right)^{N-x-1},
$$

where

$$
f_{k}=\left(\begin{array}{l}
\Omega \\
k
\end{array}\right)\left(\frac{\beta}{\alpha}\right)^{k}
$$

To evaluate the continuum limit it is convenient to write $f_{k}^{x}$ in the form

$$
f_{k}^{x}=\left[\frac{1}{\Omega^{k}}\left(\begin{array}{c}
\Omega \\
k
\end{array}\right)\right]^{\frac{t}{a}} e^{\frac{k t}{a} \ln \frac{\Omega \beta}{\alpha}}
$$

which immediately shows that assuming $\alpha=c \Omega, \beta=c \eta^{2}$ with $c$ arbitrary, we have

$$
\lim _{a \rightarrow 0} f_{k}^{x}=\delta_{k, 0}+\delta_{k, 1} e^{-2 E t} .
$$

Therefore we get the correct continuum limit for the $n=1$ two-point correlation functions, while all the correlation functions of higher powers of the composite vanish.

It is also worth while noticing the form of the wave operator appearing in this action

$$
-A=-\left[\left(\Omega+\eta^{2}\right) 1-\eta^{2} a \nabla\right],
$$

which is static and of sign opposite to that of a true nonrelativistic boson, but, as already stated, the propagator of the Cooper pair is not its inverse . 


\section{A quadratic action for the pions}

The investigation of the pairing model has confirmed our conjecture about the existence of a free action for fermionic composites, and it has also shown that its parameters are not related in a simple way to those of the constituents. In particular we have seen the explicit appearance of the index of nilpotency among the parameters. In constructing an action for the pions we will keep in mind these results. The other relevant ingredient for our problem is obviously the chiral invariance.

The composites with the quantum numbers of the pions are

$$
\vec{\pi}=i a^{2} \bar{\lambda} \gamma_{5} \vec{\tau} \lambda
$$

In the above definition the $\tau_{k}$ 's are the Pauli matrices and the sum over the colour, isospin and spin indices $a, f$ and $\beta$ of the quark field $\lambda_{f, \beta}^{a}$ is understood. The power of the lattice spacing has been introduced in order to give the fields the canonical dimension of a boson. To formulate a chiral invariant model we need also the field

$$
\sigma=a^{2} \bar{\lambda} \lambda
$$

All these composites have [1] index of nilpotency $\Omega=24$. The nilpotency however is not the only feature which distinguishes these Grassmann variables from ordinary variables. Indeed, they are not independent from one another, because the monomials

$$
\Phi_{n_{0}, n_{1}, n_{1}, n_{3}}=\sigma^{n_{0}} \pi_{1}^{n_{1}} \pi_{2}^{n_{2}} \pi_{3}^{n_{3}}
$$

vanish when the sum of the exponents $\sum_{k=0}^{3} n_{k}$ is larger than $\Omega$. Moreover, if it is equal to $\Omega$, these monomials vanish if at least one of the exponents is odd.

The chiral transformations over the quarks

$$
\begin{aligned}
\delta \lambda & =\frac{i}{2} \gamma_{5} \vec{\tau} \cdot \vec{\alpha} \lambda \\
\delta \bar{\lambda} & =\frac{i}{2} \bar{\lambda} \gamma_{5} \vec{\tau} \cdot \vec{\alpha}
\end{aligned}
$$

induce $O(4)$-transformations over the mesons

$$
\begin{aligned}
& \delta \sigma=\vec{\alpha} \cdot \vec{\pi} \\
& \delta \vec{\pi}=-\vec{\alpha} \sigma .
\end{aligned}
$$


A quadratic action with the above symmetry must therefore be of the form

$$
S_{C}=-a^{-2}\left[\frac{1}{2}(\vec{\pi}, A \vec{\pi})+\frac{1}{2}(\sigma, A \sigma)+(m, \sigma)\right],
$$

where a breaking term has been included. The scalar product is defined as

$$
(f, g)=a^{4} \sum_{x} f(x) g(x) .
$$

The powers of the lattice spacing $a$ have been introduced assuming the waveoperator $A$ to be dimensionless. The analogy with the model of ref. [5] and the pairing model suggest a form of the type

$$
A=\rho^{2}+a^{2} \square,
$$

where $\square$ is the Euclidean D'Alambertian on the lattice and the parameter $\rho$ should be such that $A$ be positive definite, namely $\rho^{2}>16$. We will, however, choose the more convenient expression

$$
A=\frac{\rho^{4}}{-a^{2} \square+\rho^{2}}
$$

which is equal to the previous one for small $a^{2}$ provided that $\rho$ is independent of $a$. With such a definition the positivity of $A$ requires only the positivity of $\rho^{2}$.

We will however consider also the case of $\rho$ and $m$ dependent from the index of nilpotency $\Omega$ and the lattice spacing $a$ according to

$$
\begin{aligned}
\rho & =\left(a M_{3}\right)^{\beta-\alpha} \\
m & =\Omega^{\gamma}\left(a M_{1}\right)^{\alpha} M_{2}
\end{aligned}
$$

where the $M_{i}$ 's are independent from the lattice spacing and the index of nilpotency.

The first two terms in the action (3.8) have dimension 6 and we are therefore free to add them to the QCD action if $\rho$ is idependent of $a$. The symmetry breaking term has instead dimension 4 (indeed it is of the same form of the quark mass term already present in the QCD action) but it formally vanishes in the continuum limit provided

$$
\alpha>0
$$


in which case it can also be added to the QCD action.

Let us make clear that the partition function is

$$
Z_{C}=\int[d \bar{\lambda} d \lambda] \exp \left(-S_{C}\right)
$$

Here and in the following differentials appearing in square brackets are understood as the product in a given order of the differentials over all the lattice sites and intrinsic quantum numbers.

\section{Breaking of the chiral invariance}

In order to investigate our model we introduce the Stratonovich-Hubbard transform [7]

$$
\begin{aligned}
\exp \left(-S_{C}\right)= & \frac{1}{(\operatorname{det} A)^{2}} \int\left[\frac{d(a \vec{\chi})}{\sqrt{2 \pi}}\right]\left[\frac{d\left(a \chi_{0}\right)}{\sqrt{2 \pi}}\right] \exp \left[-\frac{1}{2 a^{2}}\left(\vec{\chi}, A^{-1} \vec{\chi}\right)\right. \\
& \left.-\frac{1}{2 a^{2}}\left(\chi_{0}, A^{-1} \chi_{0}\right)+\frac{1}{a^{2}}(\vec{\chi}, \vec{\pi})+\frac{1}{a^{2}}\left(m+\chi_{0}, \sigma\right)\right] .
\end{aligned}
$$

The partition function can then be written

$$
\begin{aligned}
Z_{C}= & \frac{1}{(\operatorname{det} A)^{2}} \int\left[\frac{d \vec{\chi}}{\sqrt{2 \pi}}\right]\left[\frac{d \chi_{0}}{\sqrt{2 \pi}}\right] \exp \left[-\frac{1}{2 a^{2}}\left(\vec{\chi}, A^{-1} \vec{\chi}\right)-\frac{1}{2 a^{2}}\left(\chi_{0}, A^{-1} \chi_{0}\right)\right] \\
& \prod_{x}\left[D\left(\vec{\chi}, \chi_{0}\right)\right]^{\Omega / 2}
\end{aligned}
$$

where

$$
D\left(\vec{\chi}, \chi_{0}\right)=a^{2}\left[\left(m+\chi_{0}\right)^{2}+\vec{\chi}^{2}\right] .
$$

The effective action for the $\chi$ fields is therefore

$$
S_{\chi}=\frac{1}{2 a^{2}}\left[\left(\vec{\chi}, A^{-1} \vec{\chi}\right)+\left(\chi_{0}, A^{-1} \chi_{0}\right)\right]-\frac{\Omega}{2 a^{4}}\left(1, \ln D\left(\vec{\chi}, \chi_{0}\right)\right) .
$$

This action is $O(4)$ invariant for vanishing quark mass and reminds us of the Gell-Mann-Lévy model [8].

Since $\Omega$ is a rather large number we can apply the saddle-point method and evaluate the partition function as a series in inverse powers of this parameter. The saddle point equations are

$$
\begin{aligned}
\frac{\partial S_{\chi}}{\partial \chi_{0}(x)} & =a^{2}\left[\left(A^{-1} \chi_{0}\right)(x)-\Omega \frac{\chi_{0}(x)+m}{D(x)}\right]=0 \\
\frac{\partial S_{\chi}}{\partial \chi_{h}(x)} & =a^{2}\left[\left(A^{-1} \chi_{h}\right)(x)-\Omega \frac{\chi_{h}(x)}{D(x)}\right]=0 .
\end{aligned}
$$


At constant fields the minima are characterized by the parameter

$$
A_{0}=\sum_{x} A_{x y}=\rho^{2} .
$$

The minimum is achieved for $\vec{\chi}=0$ and

$$
\overline{\chi_{0}}=\frac{-m a \pm \sqrt{m^{2} a^{2}+4 \Omega \rho^{2}}}{2 a},
$$

where the $+($ respectively - ) sign has to be chosen when $m>0$ (respectively $m<0$ ), which we shall assume to be the case.

Now we assume

$$
(a m)^{2}<<4 \Omega \rho^{2}
$$

so that

$$
\overline{\chi_{0}} \approx a^{-1}\left[\sqrt{\Omega} \rho-\frac{1}{2} a m\right] .
$$

In order to keep the two terms in the r.h.s. of the previous equation of the same order in $\Omega$ we require for the exponent $\gamma$ that appears in (3.13)

$$
\gamma=\frac{1}{2}
$$

The second derivatives of $S_{\chi}$ at the minimum are

$$
\begin{aligned}
\left.\frac{\partial^{2} S_{\chi}}{\partial \chi_{0}(x) \partial \chi_{0}(y)}\right|_{\chi=\bar{\chi}} & =a^{2}\left[A_{x y}^{-1}+\delta_{x y} \frac{\Omega}{D}\right] \\
& =\frac{a^{4}}{\rho^{4}}\left(-\square+\frac{2 \rho^{2}}{a^{2}}-m \frac{\rho}{a \sqrt{\Omega}}\right) \\
\left.\frac{\partial^{2} S_{\chi}}{\partial \chi_{h}(x) \partial \chi_{k}(y)}\right|_{\chi=\bar{\chi}} & =\delta_{h k} a^{2}\left[A_{x y}^{-1}-\delta_{x y} \frac{\Omega}{D}\right] \\
& =\delta_{h k} \frac{a^{4}}{\rho^{4}}\left(-\square+m \frac{\rho}{a \sqrt{\Omega}}\right) .
\end{aligned}
$$

The propagator of the pion field to leading order

$$
\begin{aligned}
<\pi_{h}(x) \pi_{k}(y)> & =\frac{1}{Z_{C}} \frac{\Omega^{2}}{(\operatorname{det} A)^{2}} \int\left[\frac{d \chi_{0}}{\sqrt{2 \pi}}\right]\left[\frac{d \vec{\chi}}{\sqrt{2 \pi}}\right] \frac{\chi_{h}(x)}{D(x)} \frac{\chi_{k}(y)}{D(y)} \exp \left(-S_{\chi}\right) \\
& =\frac{1}{a^{4}}\left(\frac{1}{-\square+m_{\pi}^{2}}\right)_{x, y}, \quad \text { for } x \neq y
\end{aligned}
$$


turns out to have the canonical form. Its mass is therefore

$$
m_{\pi}^{2}=\frac{1}{\sqrt{\Omega} a} m \rho=\left(\frac{M_{1}}{M_{3}}\right)^{\alpha} M_{2} M_{3}\left(a M_{3}\right)^{\beta-1} .
$$

The mass of the $\sigma$-field is instead

$$
m_{\sigma}^{2}=2 a^{-2} \rho^{2}-m_{\pi}^{2}=2 a^{-2}\left(a M_{3}\right)^{2(\beta-\alpha)}-m_{\pi}^{2} .
$$

As a consequence of the inequality (4.8) $m_{\sigma}>>m_{\pi}$.

The $\sigma$-field acquires the nonvanishing expectation value

$$
<\sigma>=\frac{1}{V} \frac{\partial}{\partial\left(a^{2} m\right)} \log Z_{C} \approx \frac{a^{-2} \Omega}{\overline{\chi_{0}}+m} \approx a^{-1} \rho^{-1} \sqrt{\Omega} .
$$

We may notice how PCAC is realized in the present model. By using the chiral transformations we get the relation

$$
\frac{\partial S_{C}}{\partial \vec{\pi}} \delta \vec{\pi}+\frac{\partial S_{C}}{\partial \sigma} \delta \sigma=0
$$

which implies

$$
\sum_{y} \sigma(x) A_{x y} \vec{\pi}(y)-\vec{\pi}(x) A_{x y} \sigma(y)=m \vec{\pi}(x) .
$$

In the formal continuum limit

$$
A \approx \rho^{2}+a^{2} \square
$$

the previous identity becomes

$$
a^{2}[\sigma \square \vec{\pi}-\vec{\pi} \square \sigma]=m \vec{\pi} .
$$

The lhs of the above equation is proportional to the divergence of an axial current, namely

$$
\overrightarrow{\mathcal{A}}=\sigma \nabla \vec{\pi}-\vec{\pi} \nabla \sigma
$$

so that the previous equation can be rewritten as

$$
a^{2} \nabla \overrightarrow{\mathcal{A}}=m \vec{\pi} .
$$

This identity implies the relation

$$
a^{2} m_{\pi}^{2}\langle\sigma\rangle=m
$$




\section{The $\Omega$-expansion}

In order to formulate the $\Omega$-expansion it is convenient to introduce the fields which correspond to rescaled fluctuations

$$
\begin{aligned}
& \theta_{0}=\rho^{-2}\left(\chi_{0}-\bar{\chi}_{0}\right), \\
& \theta_{k}=\rho^{-2} \chi_{k} .
\end{aligned}
$$

In terms of these fields the function $D$ takes the form

$$
D=C^{2} F
$$

where

$$
\begin{aligned}
C & =\sqrt{\Omega \rho^{2}+\frac{1}{4} a^{2} m^{2}}+\frac{1}{2} a m \approx \sqrt{\Omega} \rho+\frac{1}{2} a m \\
F & =1+\frac{\rho^{2}}{C} 2 a \theta_{0}+\frac{\rho^{4}}{C^{2}} a^{2}\left(\theta_{0}^{2}+\vec{\theta}^{2}\right) .
\end{aligned}
$$

Finally the expansion of the pion propagator can be evaluated by the equation

$$
\begin{aligned}
<\pi_{h}(x) \pi_{k}(y)>= & \frac{1}{Z_{C}} \frac{1}{(\operatorname{det} A)^{2}} \frac{\Omega^{2} \rho^{4}}{C^{4}} \int\left[\frac{d \theta_{0}}{\sqrt{2 \pi}}\right]\left[\frac{d \vec{\theta}}{\sqrt{2 \pi}}\right] \\
& \cdot \frac{\theta_{h}(x)}{F(x)} \frac{\theta_{k}(y)}{F(y)} \exp \left(-S_{\chi}\right) \text { for } x \neq y .
\end{aligned}
$$

By expanding the $\ln D$ we rewrite the action $S_{\chi}$ as a series

$$
S_{\chi}=\sum_{n=2}^{\infty} S^{(n)} .
$$

The term $S^{(n)}$, for $n>2$, is a homogeneous polynomial of degree $n$ in the $\theta$-fields proportional to $\rho^{2 n} a^{n} / C^{n}$. The first three terms are

$$
\begin{aligned}
S^{(2)} & =a^{4} \sum_{x} \frac{1}{2} \vec{\theta}\left(-\square+m_{\pi}^{2}\right) \vec{\theta}+\frac{1}{2} \theta_{0}\left(-\square+m_{\sigma}^{2}\right) \theta_{0} \\
S^{(3)} & =\frac{\rho^{6}}{C^{3}} a^{4} \sum_{x} \frac{1}{a}\left(\frac{2}{3} \theta_{0}^{3}-2 \theta_{0} \vec{\theta}^{2}\right) \\
S^{(4)} & =\frac{\rho^{8}}{C^{4}} a^{4} \sum_{x}-\frac{1}{2} \theta_{0}^{4}-\frac{1}{2}\left(\vec{\theta}^{2}\right)^{2}+3 \theta_{0}^{2} \vec{\theta}^{2}
\end{aligned}
$$


With our choices $S^{(n)}$ turns out to be of the order $\Omega^{-\frac{n}{2}}$, for $n>2$. We actually have then an expansion in inverse powers of $\sqrt{\Omega}$, but the first correction is of order $\Omega^{-\frac{3}{2}}$.

We can now investigate which is the dependence of $\rho$ and $m$ on the lattice spacing which ensures the renormalizability of the of $\Omega$-expansion. This requires $\rho^{2} / C$ not to diverge in the continuum limit. Since we demand also $m_{\pi}$ to be finite we get from $(4.13)$

$$
\beta=1 .
$$

As a consequence of (3.14) the mass of the $\sigma$-field is divergent (see (4.14)). It follows for $C$ the following expression

$$
C=\sqrt{\Omega}\left(a M_{3}\right)^{1-\alpha}
$$

so that for small lattice spacing

$$
\frac{\rho^{2}}{C} \sim a^{1-\alpha}
$$

which therefore implies $\alpha \leq 1$.

It is remarkable that with the choice $\alpha=1 / 2$ we get a truly free action for the pions, all the interaction terms giving vanishing contributions to their $n$-point functions. Such an action, however, cannot be used to set up a new perturbative approach to QCD because it is an operator of dimension 6 which is not accompanied by thenecessary power of the lattice spacing. For this purpose one must make a different choice of the parameters, that is $\alpha=1$, for which

$$
\rho \text { independent of } a, \quad m=\frac{\sqrt{\Omega}}{\rho} a m_{\pi}^{2},
$$

which provides an interacting renormalizable model. Indeed, while for $n>4$ all the terms $S^{(n)}$ are accompanied by a factor $a^{n-4}$ and are irrelevant, the terms $S^{(3)}$ and $S^{(4)}$ survive in the formal continuum limit. The $\theta_{0}$-field has a divergent mass $\approx 1 / a$, and it can be rescaled according to $\theta_{0} \rightarrow a \theta_{0}$, showing that $S^{(3)}$ does not diverge. The $\sigma$-field has still a divergent mass and it is unphysical.

\section{Conclusion}

Effective Grassmann actions for even composites are in general polynomials of degree equal to the index of nilpotency of the composites. Their complexity 
is due to the fact that they must generate the correlation functions of all the nonvanishing powers of the composites. One can then think that the $n=1$ two-point functions can be obtained from a much simpler action. In the case of the pairing model, where the exact action of the Cooper pair has been derived from that of the nucleons, we have indeed shown that the $n=1$ correlation functions can be generated by a quadratic action which shares the specific features of the actions of even composites previously considered, namely it is static in the formal continuum limit and of sign opposite to that of a true nonrelativistic boson. We have then considered an action of this type for the pion. It includes the $\sigma$-field in order to be invariant under $[S U(2)]_{L} \otimes[S U(2)]_{R}$ transformations over the quarks. This model exhibits the Goldstone phenomenon by reducing its symmetry to the $O(3)$ isospin invariance. In the presence of an explicit breaking linear in $\sigma$ a squared mass for the pion is generated proportional to the breaking parameter.

One can regard our results as the construction of a simple model, whose main ingredient is the compositness of the mesonic fields. This model reminds us of that of Gell-Mann-Lévy, the connection being very transparent after the Stratonovitch-Hubbard representation has been introduced.

Our main motivation, however, is not model building, but to use our action to construct a new perturbative approach to QCD. Whether this is really possible can only be established by explicit calculations which are now in progress.

In any case the present results complete the construction of free actions for composite fields, which are shown to have to large extent the properties of elementary fields. This provides the building blocks for the models with quarks of lower dimensions referred to in the Introduction.

\section{A Solution of the one-dimensional model}

In this appendix we shall solve the one dimensional model defined by the action (2.12) by following a renormalization-group strategy, that is by iterating the integration of the degrees of freedom within a scale length. For our purposes the effective action at the scale $l$ is defined by

$$
e^{S_{l}}=\prod_{x} e^{\alpha \phi^{*}(x) \phi(x)} I(x, l)
$$


where the general form of the hopping part of the interaction between the sites $x$ and $x+l$ can be taken to be

$$
I(x, l)=\sum_{k=0}^{\Omega} C_{k}(l)\left[\phi(x) \phi^{*}(x+l)\right]^{k}
$$

with $x$ running on the lattice with spacing $l$.

The thinning of the degrees of freedom amounts to the evaluation of the integral

$$
\begin{aligned}
I(x-l, 2 l)= & \int d \phi^{*}(x) d \phi(x) e^{\alpha \phi^{*}(x) \phi(x)} I(x-l, l) I(x, l) \\
= & \int d \phi^{*}(x) d \phi(x) \sum_{n=0}^{\Omega} \frac{\alpha^{n}}{n !}\left[\phi^{*}(x) \phi(x)\right]^{n} . \\
& \sum_{k=0}^{\Omega} C_{k}^{2}(l)\left[\phi^{*}(x) \phi(x) \phi(x-l) \phi^{*}(x+l)\right]^{k} \\
= & \sum_{k=0}^{\Omega} \frac{\alpha^{\Omega-k}}{(\Omega-k) !}(\Omega !)^{2} C_{k}^{2}(l)\left[\phi(x-l) \phi^{*}(x+l)\right]^{k},
\end{aligned}
$$

so that

$$
C_{k}(2 l)=\frac{\alpha^{\Omega-k}}{(\Omega-k) !}(\Omega !)^{2} C_{k}^{2}(l) .
$$

By iteration we get the solution of this recursion relation

$$
C_{k}(l)=\left[\frac{\alpha^{\Omega-k}}{(\Omega-k) !}(\Omega !)^{2}\right]^{l-1} C_{k}^{l}(1),
$$

which has the initial condition

$$
C_{k}(1)=\frac{\beta^{k}}{k !}
$$

By using periodic boundary conditions on a lattice of size $N$ we obtain for the partition function

$$
\begin{aligned}
Z & =\int d \phi^{*}(0) d \phi(0) e^{\alpha \phi^{*}(0) \phi(0)} I(0, N) \\
& =\sum_{k=0}^{\Omega} C_{k}(N) \frac{\alpha^{\Omega-k}}{(\Omega-k) !}(\Omega !)^{2}
\end{aligned}
$$




$$
\begin{aligned}
& =\sum_{k=0}^{\Omega} C_{k}^{N}(1)\left[\frac{\alpha^{\Omega-k}}{(\Omega-k) !}(\Omega !)^{2}\right]^{N} \\
& =\left(\alpha^{\Omega} \Omega !\right)^{N} \sum_{k=0}^{\Omega}\left[\left(\frac{\beta}{\alpha}\right)^{k}\left(\begin{array}{l}
\Omega \\
k
\end{array}\right)\right]^{N} \\
& =\left(\alpha^{\Omega} \Omega !\right)^{N} \sum_{k=0}^{\Omega} f_{k}^{N}
\end{aligned}
$$

where the coefficients $f_{k}$ have been defined in (2.15). Similarly for the twopoint functions we have

$$
\begin{aligned}
\left\langle\left[\phi^{*}(0) \phi(x)\right]^{n}\right\rangle= & \frac{1}{Z} \int d \phi^{*}(0) d \phi(0) d \phi^{*}(x) d \phi(x) e^{\alpha\left[\phi^{*}(0) \phi(0)+\phi^{*}(x) \phi(x)\right]} \\
& \sum_{r=0}^{\Omega} \sum_{k=0}^{\Omega-n} C_{k}(x) C_{r}(N-x)\left[\phi(0) \phi^{*}(x)\right]^{k+n}\left[\phi(x) \phi^{*}(0)\right]^{r} \\
= & \frac{1}{Z} \int d \phi^{*}(0) d \phi(0) d \phi^{*}(x) d \phi(x) e^{\alpha\left[\phi^{*}(0) \phi(0)+\phi^{*}(x) \phi(x)\right]} \\
& \sum_{k=0}^{\Omega-n} C_{k+n}(x) C_{k}(N-x)\left[\phi^{*}(0) \phi(0) \phi^{*}(x) \phi(x)\right]^{k+n} \\
= & \frac{1}{Z} \sum_{k=0}^{\Omega-n} C_{k+n}(x) C_{k}(N-x) \frac{\alpha^{\Omega-k-n}}{(\Omega-k-n) !}(\Omega !)^{2} \\
= & \left\{\sum_{k=0}^{\Omega} f_{k}^{N}\right\}^{-1} \frac{1}{\beta^{n}} \sum_{k=0}^{\Omega-n} \frac{(k+n) !}{k !}\left(f_{k+n}\right)^{x+1}\left(f_{k}\right)^{N-x-1},(\mathrm{~A}
\end{aligned}
$$

which is the result given in (2.14). 


\section{References}

[1] F. Palumbo, Phys. Rev. 48D (1993) R1917; G. De Franceschi and F. Palumbo, Modern Phys. Lett. A11 (1995) 901.

[2] N. Kawamoto and J. Smit, Nucl. Phys. B192 (1981) 100 ; H. KlubergStern, A. Morel and B. Petersson, Nucl. Phys. B215 [FS7] (1983) 527; H. Kluberg-Stern, A. Morel, O. Napoly and B. Petersson, Nucl. Phys. B190 [FS3] (1981) 504

[3] I. Barbour, N.-E. Behilil, E. Dagotto, F. Karsch, A. Moreo, M. Stone and H. W. Wyld, Nucl. Phys. B275[FS17] (1986) 296

[4] P. Rossi and U. Wolff, Nucl. Phys. B248 (1984) 105

[5] F. Palumbo, Phys. Lett. B328 (1994) 79

[6] A. M. Barbaro, A. Molinari and F. Palumbo, Nucl. Phys. B487 (1997) 492

[7] R. L. Stratonovitch, Dokl. Acad. Nauk. S.S.S.R. 115 (1957) 1097 [Sov. Phys. Dokl. 2 (1958) 416 ]; J. Hubbard, Phys. Rev. Lett. 3 (1959) 77

[8] M. Gell-Mann and M. Lévy, Nuov. Cim. 16 (1960) 705

[9] F. Palumbo, to be published 